\title{
Surface energetics and structure of the Ge wetting layer on $\mathrm{Si}(100)$
}

\author{
M. J. Beck,* A. van de Walle, and M. Asta \\ Materials Science and Engineering, Northwestern University, Evanston, Illinois 60208, USA \\ (Received 27 January 2004; revised manuscript received 2 July 2004; published 23 November 2004)
}

\begin{abstract}
Ge deposited on $\mathrm{Si}(100)$ initially forms heteroepitaxial layers, which grow to a critical thickness of $\sim 3$ MLs before the appearance of three-dimensional strain relieving structures. Experimental observations reveal that the surface structure of this Ge wetting layer is a dimer vacancy line (DVL) superstructure of the unstrained $\mathrm{Ge}(100)$ dimer reconstruction. In the following, the results of first-principles calculations of the thickness dependence of the wetting layer surface excess energy for the $c(4 \times 2)$ and $4 \times 6$ DVL surface reconstructions are reported. These results predict a wetting layer critical thickness of $\sim 3$ MLs, which is largely unaffected by the presence of dimer vacancy lines. The $4 \times 6$ DVL reconstruction is found to be thermodynamically stable with respect to the $c(4 \times 2)$ structure for wetting layers at least $2 \mathrm{ML}$ thick. A strong correlation between the fraction of total surface induced deformation present in the substrate and the thickness dependence of wetting layer surface energy is also shown.
\end{abstract}

DOI: 10.1103/PhysRevB.70.205337 PACS number(s): 68.35.Md, 68.47.Fg, 81.15.Aa, 68.35.Bs

\section{INTRODUCTION}

The self-assembly of nanostructures with technologically useful electronic and optical properties has recently led to intense interest in understanding and controlling the heteroepitaxial growth and morphology of crystalline thin films. ${ }^{1,2} \mathrm{Ge}$ on $\mathrm{Si}(100)$ heteroepitaxy has served as a model system for many theoretical and experimental investigations in this area. ${ }^{3}$ Heteroepitaxial growth of $\mathrm{Ge}$ on $\mathrm{Si}(100)$ proceeds in the Stranski-Krastanow (SK) growth mode, ${ }^{4}$ in which increasing misfit strain energy in the thickening overlayer film, the wetting layer, leads to the formation of strain relieving three-dimensional mounds, quantum dots (QDs), at the expense of increased surface energy. Recent experimental work has revealed surprising complexity in both quantum dot and wetting layer morphology. Ge quantum dots on $\mathrm{Si}(100)$, observed as (105) faceted pyramids by Mo et al., ${ }^{5}$ have been observed to develop into complex multifaceted domes. ${ }^{4,6}$ Unfaceted surface mounds have been observed by scanning tunnelling microscopy (STM), ${ }^{7}$ and their role as precursors to faceted pyramids has recently been investigated. ${ }^{8,9}$ Experiments by Sutter and Lagally ${ }^{10,11}$ and Tromp et al. ${ }^{12}$ have also demonstrated that given appropriate growth conditions faceted pyramids can arise continuously from surface ripples.

Substantial effort has been dedicated to developing continuum theories for modeling the self-assembly of QD islands in heteroepitaxial SK growth. To reproduce the observed formation of a wetting layer with a highly uniform thickness measured in atomic monolayers (MLs), and the wetting layer's stability subsequent to the formation of QD islands, these theories include a "wetting potential,"13 which parametrizes the nonlinear thickness dependence ${ }^{14-18}$ of the surface excess energy of a coherently strained overlayer film. Recently, Golovin, Davis, and Voorhees ${ }^{13}$ have demonstrated that variations in the detailed form of the wetting potential can have profound consequences on the morphology and spatial distribution of QD island arrays in SK growth. These results provide a strong motivation for employing firstprinciples calculations to characterize the detailed nature and origin of the thickness dependence of the wetting layer excess energy in common SK systems, such as Ge on $\mathrm{Si}(100)$.

Unstrained $\mathrm{Si}$ and $\mathrm{Ge}(100)$ surfaces reconstruct by forming bonded surface atom pairs, or dimers, which align in a (110) direction to form dimer rows. Calculations and experimental results have demonstrated that tilting the dimer bonds in various patterns reduces the total surface energy. For Ge, calculations reveal that the $c(4 \times 2)$ reconstruction, in which the dimer bond tilts alternate along and perpendicular to the dimer rows, and the $p(2 \times 2)$ reconstruction, in which dimer bond tilts alternate only perpendicular to the dimer rows, lie extremely close in energy, ${ }^{19-21}$ and thus both reconstructions are expected to be observed at finite temperature.

Higher order variants of these dimer structures have been observed to occur in the $\mathrm{Ge}$ on $\mathrm{Si}(100)$ wetting layer surface. ${ }^{7,22,23}$ These structures involve periodic arrangements of ordered dimer vacancies, or dimer vacancy lines (DVLs). A $4 \times N$ (or $2 \times N)$ DVL reconstruction results from a $c(4 \times 2)(p(2 \times 2)$ or $2 \times 1)$ dimer reconstructed surface with every $N$ th dimer column removed (see Fig. 1). ${ }^{22}$ More complex $M \times N$ patchlike reconstructions ${ }^{7,23}$ have been observed in high resolution STM studies. Each succeeding ML of deposited Ge has dimer bonds, and therefore DVLs, perpendicular to those of the preceding layer. As such, the $M \times N$ patch structure is described as an $M \times 4$ layer atop a $4 \times N$ layer, for which the upper layer does not fill in the preceding layer DVLs. Moreover, recent work ${ }^{24,25}$ indicates that nontrivial intermixing is expected to occur across the $\mathrm{Ge}-\mathrm{Si}$ interface.

Complete characterization of the energetics of the $\mathrm{Ge}$ on $\mathrm{Si}(100)$ wetting layer system requires consideration of the wide array of complex structural features observed to date. At present, a study involving the most accurate firstprinciples calculations and incorporating the full range of possible surface reconstructions as well as arbitrary intermixing is intractable. Important insights into the nature and origin of $\mathrm{Ge}$ on $\mathrm{Si}(100)$ wetting layer structures may nonetheless be gathered from studies considering only various subsets of the observed reconstructions, as previously demonstrated. ${ }^{26-33}$ 


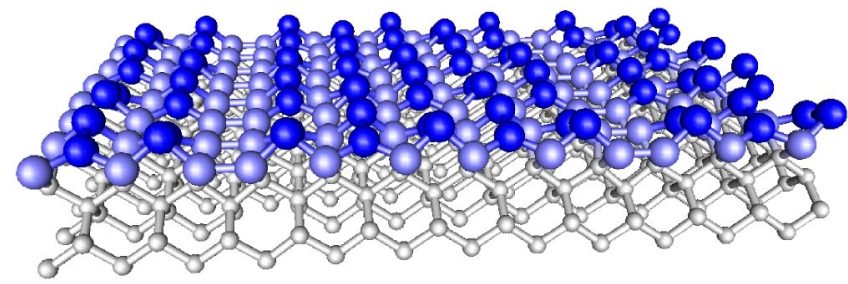

FIG. 1. (Color online) Schematic showing a two layer $4 \times 6$ DVL Ge wetting layer on $\mathrm{Si}(100)$. Surface Ge atoms are darker than second layer $\mathrm{Ge}$ atoms to highlight underlying $c(4 \times 2)$ tilted dimer structure, clearly visible at right, and second layer Ge-Ge rebonding. Dimer bonds, and therefore dimer vacancy lines, run into and out of the page.

Using a modified Keating model, Tersoff calculated ${ }^{26}$ the chemical potential of $\mathrm{Ge}$ atoms in a dimer reconstructed $\mathrm{Ge}$ wetting layer on $\mathrm{Si}(100)$ as a function of thickness. Estimating the limits of the chemical potential of Ge atoms in established QD islands, he extracted a critical Ge wetting layer thickness of 3 ML. Further, it was demonstrated that a nontrivial wetting layer critical thickness results from misfit strain in combination with the difference in stiffness between $\mathrm{Ge}-\mathrm{Ge}$ and $\mathrm{Si}-\mathrm{Si}$ bonds. Tersoff also performed analogous calculations $^{27}$ to study the $2 \times N$ DVL reconstructed surface and demonstrated that the compressive misfit strain in the Ge on $\mathrm{Si}(100)$ wetting layer stabilizes rebonding in the second layer atoms beneath the dimer vacancies, and that this rebonding, which reduces the number of surface dangling bonds, is essential in stabilizing the overall $2 \times N$ DVL reconstruction.

Further work has cemented the initial picture of misfit strain stabilized rebonding of the exposed second layer atoms as the mechanism which leads to the formation of the $4 \times N$ DVL versus the $c(4 \times 2)$ reconstructed wetting layer surface. The formation energy of isolated dimer vacancies has been calculated and is found to be negative for $\mathrm{Ge}$ on $\mathrm{Si}(100)$ given appropriate second layer atom rebonding. It has been established that interactions between individual dimer vacancies lead to an ordering into dimer vacancy lines, ${ }^{28,29}$ and the selection of a regular DVL spacing. ${ }^{30}$ Lagally and coworkers have demonstrated that experimental results for $2 \times N$ DVL reconstructed wetting layers are consistent with only limited Ge-Si intermixing. ${ }^{30}$ Using an elasticity model, DVL-DVL interaction parameters have been extracted from experiment. ${ }^{31}$ The DVL spacing dependence of surface energy has been investigated from first principles in single layer $2 \times N$ DVL Ge on $\mathrm{Si}(100)$ structures. ${ }^{32,33}$ A recent study using the Tersoff potential has examined the energetics of the $2 \times N$ DVL wetting layer surface as a function of thickness and $N$ vacancy line spacing. ${ }^{29}$

In the following we report the results of first-principles total energy calculations of the Ge wetting layer on $\mathrm{Si}(100)$ energetics as a function of wetting layer thickness and surface structure. These results are a direct parametrization of the wetting potential for a subset of the observed DVL reconstructions in the $\mathrm{Ge}$ on $\mathrm{Si}(100)$ system. We examine pure Ge films taking either the alternating tilted dimer $c(4 \times 2)$ or the $4 \times 6$ DVL reconstructed surface structure, as an exhaustive survey of the full range of the observed wetting layer surface reconstructions including intermixing is not presently tractable from first principles. Calculations on this subset provide additional insight into the wetting layer structure and energetics, and our results form the basis for an analysis of the overall factors governing the form of the wetting layer excess energy thickness dependence.

\section{METHOD}

First-principles total energy calculations were performed within electronic density functional theory and the local density approximation using the $a b$ initio total-energy and molecular-dynamics program VASP (Vienna Ab Initio Simulation Program) developed at the Institut für Materialphysik of the Universität Wien. ${ }^{34-37}$ Exchange and correlation were described by the Ceperley-Alder functional ${ }^{38}$ as parametrized by Perdew and Zunger, ${ }^{39}$ and core-electron interactions were modeled using Vanderbilt ultrasoft pseudopotentials. ${ }^{40,41}$ All calculations, including reference calculations discussed below, were conducted with the same plane wave kinetic energy cutoff, total energy and residual force convergence criterion, and using equivalent $k$-point meshes. ${ }^{42}$

Surface energies were calculated using periodic boundary conditions and a repeated slab geometry. We considered twocomponent slabs consisting of a Ge wetting layer atop an unstrained $\mathrm{Si}(100)$ substrate. The Ge wetting layer surface was either $c(4 \times 2)$ reconstructed, or, as representative of the family of $4 \times N$ DVL structures, $4 \times 6$ DVL reconstructed. The bottoms of these $\mathrm{Ge}$ on $\mathrm{Si}(100)$ wetting layer slabs were terminated with a $\mathrm{Si} c(4 \times 2)$ reconstructed surface. Unless otherwise noted, all slabs contained this identical substrate. The unstrained bulk Si lattice parameter $(5.39 \AA)$ was calculated in a reference calculation by minimizing the total energy of a single Si bulk cubic unit cell with respect to lattice constant. Wetting layer-substrate intermixing was not considered, and the Ge-Si interface was coherent.

Calculation parameters were chosen such that calculated absolute surface energies are estimated to be converged to within $\sim 2 \mathrm{meV} / \AA^{2}$ and surface energy differences between films with varying thickness and/or surface reconstructions are converged to within $\sim 0.1 \mathrm{meV} / \AA^{2}$. For all calculations the electronic wave function was expanded in plane waves with a kinetic energy cutoff of $188.2 \mathrm{eV}$ (13.8 Ryd), and reciprocal space summations were performed using a Monkhorst-Pack mesh ${ }^{43}$ with a density of $8 \times 6 k$ points per reciprocal $1 \times 1$ surface cell. The $k$-point density along the reciprocal lattice direction normal to the slab surface was chosen as $1 k$-point per reciprocal lattice vector.

Energy convergence was also checked with respect to slab and vacuum thickness to ensure that surface-surface interactions through the slab and between slabs were minimal, and that residual forces on the fixed atoms were small. This last condition was found to be the most restrictive, and in order to satisfy the convergence criterion, the Si substrate thickness was chosen as $16 \mathrm{MLs}$, and the vacuum separation of slabs was set to $\sim 28 \AA$. The positions of atoms in the center two layers of the substrate were not allowed to relax, while the positions of all other atoms were allowed to relax according to the calculated ionic forces until the total energy of the 


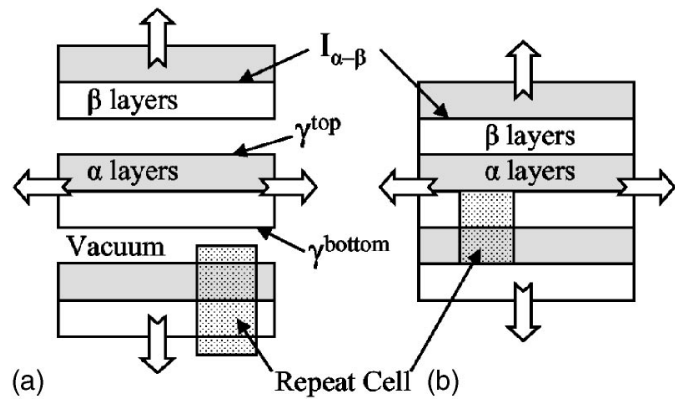

FIG. 2. Schematic of two-component slab geometries employed in calculations of surface energies (a) and interface energies (b). In $\mathrm{Ge}$ on $\mathrm{Si}(100)$ wetting layer slabs, $\alpha$ layers are $\mathrm{Ge}$ and $\beta$ layers are Si substrate layers. $\gamma^{\text {top }}$ is then $\gamma^{\mathrm{WL}}$, and $I_{\alpha-\beta}$ is $I_{\mathrm{WL}-\text { substrate }}$, where $\Gamma^{\mathrm{WL}}$ is the sum of these excess energies.

repeat cell was converged to within $10^{-5} \mathrm{eV}$. Slabs of this geometry have at least seven fully relaxed layers associated with each surface of interest. The residual forces on all atoms after full relaxation were less than $0.05 \mathrm{eV} / \AA$.

\section{THERMODYNAMIC MODEL}

\section{A. Surface Energies}

The calculated total energy of a single-component repeated slab per unit cell may be written as

$$
E=n e+2 A \gamma,
$$

for $n$ the number of atoms per repeat cell, $A$ the surface area of one side of the slab, with the factor of two correcting for the two slab surfaces per repeat cell, and $e$ the reference energy of a single atom in the equilibrium bulk configuration. The surface excess energy per unit surface area $\gamma$ is unique to a given surface structure. A multicomponent slab with different atomic species segregated by layer [see Fig. 2(a)] may be treated similarly with

$$
E=\sum_{\alpha} n_{\alpha} e_{\alpha}+A\left(\gamma^{\text {top }}+\gamma^{\text {bottom }}+\sum_{\alpha-\beta} I_{\alpha-\beta}\right),
$$

where subscripts indicate atomic species. $I_{\alpha-\beta}$ is the excess energy of an interface between layers of species $\alpha$ and $\beta$, and structures with different top and bottom surfaces (structurally, compositionally, or both) are allowed with the inclusion of separate $\gamma$ terms. For a two component slab we may extract $I_{\alpha-\beta}$ explicitly from a system of alternating regions of $\alpha$ and $\beta$ layers [see Fig. 2(b)]. Here,

$$
E=n_{\alpha} e_{\alpha}+n_{\beta} e_{\beta}+2 A I_{\alpha-\beta} .
$$

Combined with a calculation in this geometry, $I_{\alpha-\beta}$ and $\gamma^{\text {top }}$ $+\gamma^{\text {bottom }}$ may be derived from the total excess energy of a two component slab structure.

Consider now a Ge wetting layer atop a $\mathrm{Si}(100)$ substrate, as in Fig. 2(a), where the total energy of the repeat cell is written as in Eq. (1). As noted above we consider cells with identical substrate dimensions and configurations, and therefore may define a constant $E_{\text {substrate }}=n_{\mathrm{Si}} e_{\mathrm{Si}}+A \gamma^{\text {substrate }}$. Combining the wetting layer surface energy and the wetting layer- substrate interface energy as $\Gamma^{\mathrm{WL}}=\gamma^{\mathrm{WL}}+I_{\mathrm{WL}-\text { substrate, }}$, we may rewrite Eq. (1) as

$$
E=E_{\text {substrate }}+n_{\mathrm{Ge}} e_{\mathrm{Ge}}+A \Gamma^{\mathrm{WL}} .
$$

In this form it is clear that the relative stability of two systems containing identical $n_{\mathrm{Ge}}$ but having different wetting layer surface structures is simply determined by comparing $\Gamma^{\mathrm{WL}}$, the total wetting layer excess energy.

\section{B. Surface Phase Stability}

For a given $n_{\mathrm{Ge}}$ and $A$ the relative stability of various surface reconstructions may be determined from Eq. (2) by comparing the calculated $\Gamma^{\mathrm{WL}}$ for each surface phase. Thus we effectively compare the excess energy per atom of the wetting layer. Different surface phases, e.g., the $c(4 \times 2)$ and $4 \times N$ DVL reconstructions studied here, have different areal densities of surface atoms. Thus, selecting arbitrary $n_{\mathrm{Ge}}$ and $A$ requires considering slabs with different, and even fractional, wetting layer thicknesses. The geometry of the repeat cells for the present first principles calculations require that we calculate $\Gamma^{\mathrm{WL}}$ for integer wetting layer thickness, that is, only complete layer structures with uniform thickness. In order to examine the relative stability of the $4 \times 6$ DVL surface reconstruction and the $c(4 \times 2)$ tilted dimer reconstruction we must interpolate $\Gamma^{\mathrm{WL}}\left(n_{\mathrm{Ge}}\right)$ for arbitrary $n_{\mathrm{Ge}}$, and therefore for arbitrary wetting layer thicknesses.

Defining $n_{\text {surf }}$ and $n_{\text {bulk }}$ as the number of atoms per unit area in, respectively, the surface layer and a nonsurface (bulklike) layer, we have $n_{\text {surf }}=n_{\text {bulk }}$ for the $c(4 \times 2)$ reconstruction, and $n_{\text {surf }}=[(N-1) / N] n_{\text {bulk }}$ for a $4 \times N$ DVL reconstruction. As $N \rightarrow \infty$, that is, as the DVL spacing approaches infinity, we recover the $c(4 \times 2)$ reconstruction. Regardless of surface reconstruction, $n_{\text {bulk }}$ is a constant fixed by the bulk structure and repeat cell dimensions. The number of complete nonsurface (bulklike) layers in a wetting layer containing $n_{\mathrm{Ge}}$ total atoms per unit area terminated by a surface phase with $n_{\text {surf }}$ atoms in the surface layer is the maximum integer $l$, where

$$
l \leqslant \frac{n_{\mathrm{Ge}}-n_{\mathrm{surf}}}{n_{\text {bulk }}} .
$$

When $l=\left(n_{\mathrm{Ge}}-n_{\text {surf }}\right) / n_{\text {bulk }}$ we have a uniform thickness throughout the slab, and, numbering up from the wetting layer-substrate interface, we have $l$ complete bulklike layers, and layer $l+1$ is the surface layer containing $n_{\text {surf }}$ atoms. We distinguish the properties of these complete layer slabs with a subscripted notation, where, for example, the wetting layer excess energy is $\Gamma_{l+1}^{\mathrm{WL}}$. That is, for a slab with $n_{\mathrm{Ge}}, n_{\text {bulk }}$, and $n_{\text {surf }}$ such that the equality holds in Eq. (3), $\Gamma^{\mathrm{WL}}\left(n_{\mathrm{Ge}}\right)$ $\equiv \Gamma_{l+1}^{\mathrm{WL}}$. For cases where $l<\left(n_{\mathrm{Ge}}-n_{\text {surf }}\right) / n_{\text {bulk }}$ we model the wetting layer as having two regions of different thickness. Part of the film is as described, but the remaining part has $l+1$ bulk layers and a surface layer numbered $l+2$. The $l$ +1 layer thick region, or terrace, is separated from the $l+2$ layer thick region, or terrace, by a single-layer height step whose energy and interactions we neglect. The surface reconstruction itself is identical on each terrace. Increasing the 
wetting layer thickness by one ML uniformly over the entire surface while retaining the same surface phase requires the addition of $n_{\text {bulk }}$ Ge atoms regardless of the value of $n_{\text {surf }}$ imposed by the surface structure, or whether the slab is of uniform thickness or has regions of different thickness.

Throughout this work we treat DVLs as components of the surface reconstruction itself, not as structures composed of steps on the $c(4 \times 2)($ or $p(2 \times 2))$ reconstructed surface. DVLs do not alter the overall thickness of the wetting layer, and their spacing is fixed as $N$. The single-height step separating terraces of thickness $l+1$ and $l+2$ discussed above is entirely distinct from a DVL, and may have arbitrary spacing. The thermodynamic construct employed here is therefore exact for the case where the energetics and interactions of the steps separating the different thickness terraces are neglected-an approximation appropriate in the limit of infinite sized $l+1$ and $l+2$ layer thick terraces.

Given first-principles results $\Gamma_{l+1}^{\mathrm{WL}}$ and $\Gamma_{l+2}^{\mathrm{WL}}$, the surface excess energy for a wetting layer $l+1$ and $l+2$ layers thick, respectively, we may interpolate $\Gamma^{\mathrm{WL}}\left(n_{\mathrm{Ge}}\right)$ within the above thermodynamic construct, for

$$
n_{\text {surf }}+n_{\text {bulk }} l<n_{\mathrm{Ge}}<n_{\text {surf }}+n_{\text {bulk }}(l+1),
$$

following Eq. (2) as

$$
\begin{aligned}
\Gamma^{\mathrm{WL}}\left(n_{\mathrm{Ge}}\right)= & \left(\frac{n_{\mathrm{Ge}}-n_{\text {surf }}}{n_{\text {bulk }}}-l\right) \Gamma_{l+2}^{\mathrm{WL}} \\
& +\left[1-\left(\frac{n_{\mathrm{Ge}}-n_{\text {surf }}}{n_{\text {bulk }}}-l\right)\right] \Gamma_{l+1}^{\mathrm{WL}} .
\end{aligned}
$$

Thus, $\Gamma^{\mathrm{WL}}\left(n_{\mathrm{Ge}}\right)$, the surface excess energy for a wetting layer with a particular surface reconstruction but containing an arbitrary number of $\mathrm{Ge}$ atoms, is simply the weighted linear combination of $\Gamma_{l+1}^{\mathrm{WL}}$ and $\Gamma_{l+2}^{\mathrm{WL}}$ for $l$ determined as above. The weights of $\Gamma_{l+1}^{\mathrm{WL}}$ and $\Gamma_{l+2}^{\mathrm{WL}}$ are simply the fraction of the total surface area of the wetting layer with layer thickness $l+1$ and $l+2$, respectively. If we plot a set of calculated $\Gamma_{l+1}^{\mathrm{WL}}$ versus $n_{\mathrm{Ge}}$ for a particular surface phase, $\Gamma^{\mathrm{WL}}\left(n_{\mathrm{Ge}}\right)$ for arbitrary $n_{\mathrm{Ge}}$ may be interpolated as the lines connecting neighboring $\Gamma_{l+1}^{\mathrm{WL}}$ points. This follows directly from Eq. (4) for a plot of $\Gamma_{l+1}^{\mathrm{WL}}$ with $n_{\mathrm{Ge}}$ on the abscissa. Furthermore, if we plot sets of $\Gamma_{l+1}^{\mathrm{WL}}$ versus $n_{\mathrm{Ge}}$ for various surface phases on the same plot and construct the convex hull of the data with lines connecting neighboring $\Gamma_{l+1}^{\mathrm{WL}}$ points-regardless of surface phase-we may read the evolution of the stable surface phase as a function of $n_{\mathrm{Ge}}$ (and by extension, wetting layer thickness) directly.

\section{Wetting layer critical thickness}

As a basis for determining an estimate of the wetting layer critical thickness, we employ the thermodynamic framework outlined by Tersoff. ${ }^{26}$ In this construct we compare the energy required to uniformly increase the thickness of the wetting layer by one $\mathrm{ML}$ as opposed to incorporating the same number of atoms into an existing QD island. Following the notation developed above, the energy per atom required to increase the wetting layer thickness from $l+1$ to $l+2$ total MLs is simply
TABLE I. First-principles calculated $c(4 \times 2)$ alternating tilted dimer and $2 \times 1$ flat dimer reconstructed $\mathrm{Si}$ and $\mathrm{Ge}$ (100) surface energies in $\mathrm{meV} / \AA^{2}$. Unless otherwise noted, surface energies are for structures at their calculated natural lattice constant.

\begin{tabular}{lcc}
\hline \hline & $c(4 \times 2)$ & $2 \times 1$ \\
\hline $\mathrm{Si}^{\mathrm{a}}$ & 90.01 & 98.06 \\
$\mathrm{Si}^{\mathrm{b}}$ & 88.01 & 90.50 \\
$\mathrm{Si}^{\mathrm{c}}$ & 93.62 & 96.38 \\
$\mathrm{Si}^{\mathrm{d}}$ & 95.69 & \\
$\mathrm{Ge}, \epsilon_{\mathrm{Ge}}^{\mathrm{XYa}}$ & 66.19 & 75.65 \\
$\mathrm{Ge}, \epsilon_{\mathrm{Ge}}^{\mathrm{XY}}$ & 62.42 & 65.54 \\
$\mathrm{Ge}, \epsilon_{\mathrm{Si}}^{\mathrm{XY}}$ & 66.02 & 82.81 \\
\hline \hline
\end{tabular}

${ }^{\mathrm{a}}$ This work.

${ }^{\mathrm{b}}$ Reference 19.

${ }^{\mathrm{c}}$ Reference 44 .

${ }^{\mathrm{d}}$ Reference 45 .

$$
\mu_{l+2}^{\mathrm{WL}} \equiv \frac{E_{l+2}-E_{l+1}}{n_{\mathrm{bulk}}} .
$$

For $\mu^{\mathrm{QD}}$ the energy per atom required to incorporate these same $n_{\text {bulk }}$ into existing QD islands, the critical wetting layer thickness may be defined as the largest thickness $l+1$ such that $\mu_{l+1}^{\mathrm{WL}}<\mu^{\mathrm{QD}}$.

While we calculate $\mu_{l+1}^{\mathrm{WL}}$, determining a quantitative $\mu^{\mathrm{QD}}$ requires detailed knowledge of the geometry of the representative QD islands under consideration, we estimate $\mu^{\mathrm{QD}}$ following Tersoff. We bracket $\mu^{\mathrm{QD}}$ by considering two limiting cases. In the case of small, coherent islands $\mu^{\mathrm{QD}}$ is approximated by the chemical potential of a Ge atom in a bulk crystal biaxially strained to the lattice constant of $\mathrm{Si}$ and allowed to relax fully perpendicular to the applied strain, a strain state we denote $\epsilon_{\mathrm{Si}}^{\mathrm{XY}}$. As the size of the island considered is increased, misfit strain relaxation scales as $N$, the number of atoms in the QD island, but the surface excess energy scales as $N^{2 / 3}$. The $N$ scaling of strain relaxation dominates the QD island energy for larger islands, and $\mu^{\mathrm{QD}}$ decreases. In the limiting case of a very large island, $\mu^{\mathrm{QD}}$ approaches the chemical potential of a Ge atom in a fully relaxed Ge bulk crystal at its natural lattice constant, a strain state denoted $\epsilon_{\mathrm{Ge}}^{\mathrm{XY}}$.

\section{RESULTS}

As background for our calculations of wetting layer properties, we have used the above methodology and thermodynamic formalism to calculate various properties of $\mathrm{Si}$ and $\mathrm{Ge}$ and their (100) surface reconstructions. The lattice constant of $\mathrm{Si}(\mathrm{Ge})$ is calculated as $5.39 \AA(5.62 \AA)$, and the misfit strain for Ge deposited on $\mathrm{Si}(100)$ is $-4.18 \%$. The bulk chemical potential of unstrained $\mathrm{Si}(\mathrm{Ge})$ is $-5.97 \mathrm{eV} /$ atom $(-5.19 \mathrm{eV} /$ atom $)$. The calculated surface energies of the $c(4 \times 2)$ and flat dimer $2 \times 1 \mathrm{Si}$ and $\mathrm{Ge}(100)$ reconstructions are reported in Table I and compared to values previously calculated from first principles. Values calculated in this work show reasonable agreement with previous results. 


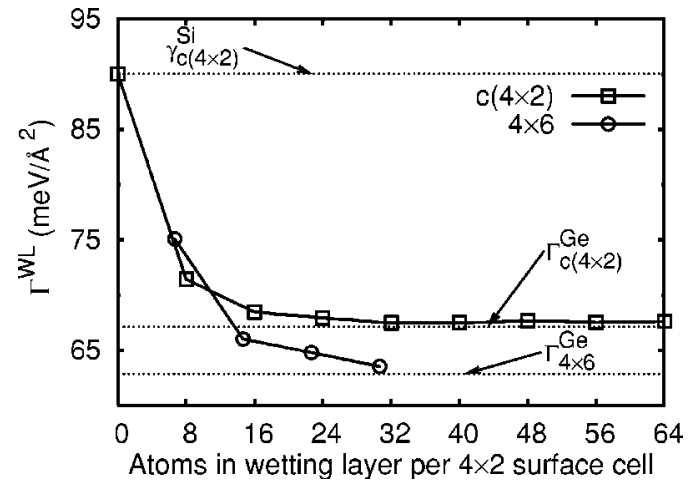

FIG. 3. $\Gamma^{\mathrm{WL}}$ as a function of $n_{\mathrm{Ge}}$, the number of Ge atoms in a $4 \times 2$ surface cell, for the $c(4 \times 2)$ alternating tilted dimer and $4 \times 6$ DVL reconstruction of a $\mathrm{Ge}$ on $\mathrm{Si}$ (100) wetting layer. The threshold values are $\gamma_{c(4 \times 2)}^{\mathrm{Si}}=90.01 \mathrm{meV} / \AA^{2}, \quad \Gamma_{c(4 \times 2)}^{\mathrm{Ge}}$ $=67.12 \mathrm{meV} / \AA^{2}$, and $\Gamma_{4 \times 6}^{\mathrm{Ge}}=62.86 \mathrm{meV} / \AA^{2}$.

Variation from previously reported values is likely due to differing slab geometries and convergence criterion, including slab thickness, vacuum spacing, plane wave kinetic energy cutoff, and reciprocal space mesh density. For comparison with the Ge on Si (100) wetting layer total excess energy, we have also calculated the pure Ge (100) $4 \times 6$ DVL surface excess energies for a Ge slab with applied strain $\epsilon=\epsilon_{\mathrm{Si}}^{\mathrm{XY}}$. The $4 \times 6$ DVL reconstructed surface has $\gamma_{4 \times 6}^{\mathrm{Ge}}\left(\epsilon_{\mathrm{Si}}^{\mathrm{XY}}\right)$ equal to $61.75 \mathrm{meV} / \AA^{2}$.

The calculated wetting layer total excess energy $\Gamma_{l+1}^{\mathrm{WL}}$ for both the tilted dimer $c(4 \times 2)$ and $4 \times 6$ DVL structures is plotted versus the number of $\mathrm{Ge}$ atoms in the wetting layer $n_{\mathrm{Ge}}$ per $c(4 \times 2)$ surface cell as points in Fig. 3 . The data points occur at $n_{\mathrm{Ge}}=n_{\text {surf }}+n_{\text {bulk }} l$, and the connecting lines are the linear interpolation of $\Gamma^{\mathrm{WL}}\left(n_{\mathrm{Ge}}\right)$ for arbitrary $n_{\mathrm{Ge}}$, following Eq. (4). Three threshold values are plotted as horizontal lines in Fig. 3. First is the bare Si (100) surface excess energy, calculated from a single component $\mathrm{Si}$ repeated slab structure as $90.01 \mathrm{meV} / \AA^{2}$. Second is $\Gamma_{c(4 \times 2)}^{\mathrm{Ge}}$ $\equiv \gamma_{c(4 \times 2)}^{\mathrm{Ge}}\left(\epsilon_{\mathrm{Si}}^{\mathrm{XY}}\right)+I_{\mathrm{Ge}-\mathrm{Si}}$, the sum of the $c(4 \times 2)(100)$ surface excess energy of a single component Ge slab strained in the plane of the surface to the Si lattice constant and the Si-Ge (100) interface excess energy. The third threshold value is $\Gamma_{4 \times 6}^{\mathrm{Ge}} \equiv \gamma_{4 \times 6}^{\mathrm{Ge}}\left(\epsilon_{\mathrm{Si}}^{\mathrm{XY}}\right)+I_{\mathrm{Ge}-\mathrm{Si}}$, the sum of the Ge $(100) 4 \times 6 \mathrm{DVL}$ surface excess energy and the Ge-Si (100) interface energy. $I_{\mathrm{Ge}-\mathrm{Si}}$ is calculated as $1.11 \mathrm{meV} / \AA^{2}, \quad \Gamma_{c(4 \times 2)}^{\mathrm{Ge}}$ as $67.12 \mathrm{meV} / \AA^{2}$, and $\Gamma_{4 \times 6}^{\mathrm{Ge}}$ as $62.86 \mathrm{meV} / \AA^{2}$. Consistent with the physical picture that any interaction between the wetting layer surface and the $\mathrm{Ge}-\mathrm{Si}$ interface should decay with increasing thickness, $\Gamma_{c(4 \times 2)}^{\mathrm{WL}}$ asymptotically approaches $\Gamma_{c(4 \times 2)}^{\mathrm{Ge}}$ as $n_{\mathrm{Ge}} \rightarrow \infty$. $\Gamma_{4 \times 6}^{\mathrm{WL}}$ shows similar behavior, but appears to converge to $\Gamma_{4 \times 6}^{\mathrm{Ge}}$ somewhat more slowly.

For wetting layer structures at least 2 MLs thick, the 4 $\times 6$ DVL surface reconstruction is stable with respect to $c(4 \times 2)$ structure. The apparent change in stability of the 4 $\times 6$ DVL and $c(4 \times 2)$ surface reconstructions between wetting layers 1 and 2 MLs thick predicted by our results is calculated for a geometry with no intermixing. Thus, the rebonded second layer atoms immediately beneath the dimer vacancies in the $4 \times 6$ DVL reconstruction are both $\mathrm{Si}$ atoms

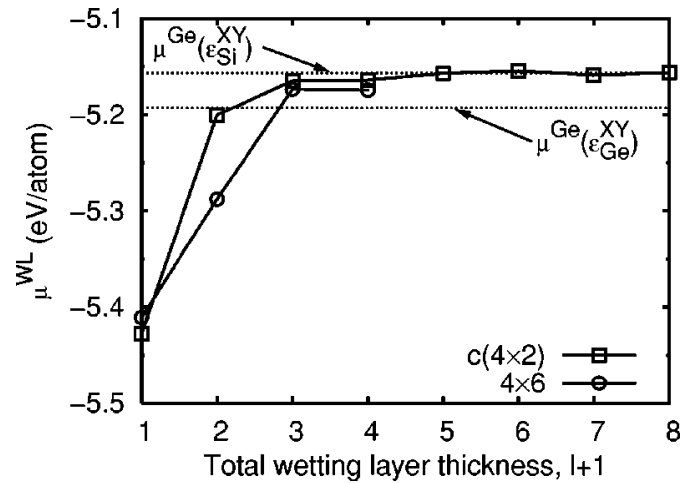

FIG. 4. Chemical potential of a Ge atom in the surface of a Ge on Si (100) wetting layer with thickness $l+1$. Dashed lines are the threshold values $\mu^{\mathrm{Ge}}\left(\epsilon_{\mathrm{Si}}^{\mathrm{XY}}\right)=-5.16 \mathrm{eV} /$ atom and $\mu^{\mathrm{Ge}}\left(\epsilon_{\mathrm{Ge}}^{\mathrm{XY}}\right)$ $=-5.19 \mathrm{eV} /$ atom, corresponding to the chemical potential of a bulk $\mathrm{Ge}$ atom biaxially strained to, respectively, the $\mathrm{Si}$ and Ge lattice constant.

in a $1 \mathrm{ML}$ wetting layer. The importance of second layer atom rebonding in determining the stability of the $4 \times N$ reconstruction has been well established, as discussed above. As $\mathrm{Si}$ is smaller than $\mathrm{Ge}$ and the $\mathrm{Si}-\mathrm{Si}$ bond is stiffer than $\mathrm{Ge}-\mathrm{Ge}$, a $\mathrm{Si}-\mathrm{Si}$ rebond would be under high tensile strain, thus having substantially higher energy than a Ge-Ge rebond. Tersoff has shown for a $1 \mathrm{ML}$ wetting layer, that replacing the rebonding atoms with Ge stabilizes the (now site specific intermixed) $4 \times N$ reconstruction. ${ }^{26}$ The results presented here show that the $4 \times 6$ DVL reconstruction with no intermixing is unstable with respect to the $c(4 \times 2)$ reconstruction for a $1 \mathrm{ML}$ wetting layer, but we anticipate that alternative $4 \times N$ DVL reconstruction geometries, e.g., those including site specific intermixing or having larger $N$ DVL spacing, may be stable.

Figure 4 plots the chemical potential of a Ge atom in the surface of the wetting layer as a function of wetting layer thickness and surface reconstruction, as described by Eq. (5). Only complete layer structures with surface layer denoted $l$ +1 are considered. An added layer to a thick enough overlayer film, regardless of surface structure, has no interaction with the buried $\mathrm{Si}-\mathrm{Ge}$ interface and behaves just as a $\mathrm{Ge}$ layer deposited on a pure Ge slab strained to $\epsilon_{\mathrm{Si}}^{\mathrm{XY}}$. The upper dashed line in Fig. 4 is this reference quantity $\mu^{\mathrm{Ge}}\left(\epsilon_{\mathrm{Si}}^{\mathrm{XY}}\right)$, calculated here as $-5.16 \mathrm{eV} /$ atom. $\mu^{\mathrm{WL}}$ converges to this value with increasing wetting layer thickness as expected.

As discussed above, $\mu^{\mathrm{Ge}}\left(\epsilon_{\mathrm{Si}}^{\mathrm{XY}}\right)$ is also the small QD island limiting value for $\mu^{\mathrm{QD}}$. The lower dashed line in Fig. 4 is the large island limiting value $\mu^{\mathrm{QD}}=\mu^{\mathrm{Ge}}\left(\epsilon_{\mathrm{Ge}}^{\mathrm{XY}}\right)$, calculated here as $-5.19 \mathrm{eV} /$ atom. The $\mu_{c(4 \times 2)}^{\mathrm{WL}}$ results compare favorably with Tersoff's results for the $2 \times 1$ non-DVL reconstruction, ${ }^{26}$ and indicate that for small (105) hut QD islands, where $\mu^{\mathrm{QD}}$ is expected to fall in the upper half of the limiting range, a wetting layer critical thickness of at least 2 MLs is expected. The comparable values of $\mu_{c(4 \times 2)}^{\mathrm{WL}}$ and $\mu_{4 \times 6}^{\mathrm{WL}}$ for thicknesses greater than 2 MLs indicates that the presence of DVLs should not strongly effect the wetting layer critical thickness. 


\section{DISCUSSION AND SUMMARY}

Examining Figs. 3 and 4 we note that the energetics of the $c(4 \times 2)$ and $4 \times 6$ DVL reconstructed wetting layers are qualitatively similar. While the $4 \times 6$ DVL reconstructed surface is clearly stable for thicker wetting layers, in both cases $\Gamma^{\mathrm{WL}}$ varies strongly with thickness for the first few monolayers. Previous results by Tersoff ${ }^{26}$ indicate that the form of the $\Gamma^{\mathrm{WL}}$ thickness dependence is a manifestation the shift of deformation due to the presence of the reconstructed wetting layer surface from the stiffer Si substrate (in thinner wetting layer structures) to the Ge overlayers (in thicker wetting layers). Following this we may analyze relaxed atomic positions resulting from our first-principles calculations to explore the physics underpinning the $\Gamma^{\mathrm{WL}}$ decay with thickness, and hence the required form of the wetting layer potential.

We extract a measure of the local deformation surrounding one atom by comparing that atom's relaxed nearest neighbor bond lengths in a particular structure with the equilibrium bulk bond lengths for the same atom type and strain state. For a structure containing a surface we extend this single atom measure of local bond deformation to construct a measure of the magnitude of local bond deformation as a function of the number of layers below the reconstructed surface. For a fully relaxed single component surface slab, consider an atom $i$ in layer $\alpha$ with nearest neighbors $j$ and define

$$
\begin{gathered}
d_{i}=\sum_{j \neq i}\left(L_{i j}^{R}-L^{\mathrm{bulk}}\right)^{2}, \\
d_{\alpha}=\sum_{i \in \alpha} d_{i} \text { and, } D^{\alpha}=\frac{d_{\alpha}}{\sum_{\alpha} d_{\alpha}} .
\end{gathered}
$$

Here, $L_{i j}^{R}$ is the calculated bond length between nearest neighbor atoms $i$ and $j$ in a fully relaxed surface slab, and $L^{\text {bulk }}$ is the bulk reference nearest neighbor distance. $L^{\text {bulk }}$ is a constant for single component systems, and may be calculated with all calculation parameters, including $k$-point mesh density, plane wave kinetic energy cutoff, and convergence criterion, conserved with respect to the associated surface slab calculation. Similarly, consider the normalized quantity

$$
G^{\alpha}=\frac{g_{\alpha}}{\sum_{\alpha} g_{\alpha}},
$$

where $g_{\alpha}=\Gamma_{\alpha+1}^{\mathrm{WL}}-\Gamma_{\alpha}^{\mathrm{WL}}$, and $\Gamma_{\alpha}^{\mathrm{WL}}$ is defined as above.

We now take $D^{\alpha}$, the relative local deformation of atoms in layer $\alpha$, of a single component slab with the $c(4 \times 2)$ surface reconstruction as an approximation of $D^{\alpha}$ for the two component wetting layer slabs. This neglects the effects of the Ge-Si interface, which are likely to be small given the coherent nature of the interface. Thus we consider the fractional reduction in wetting layer excess energy that is realized with the addition of layer $\alpha$ to the wetting layer $G^{\alpha}$ and compare directly to the fraction of the total local deformation in a slab structure, due to the presence of the wetting layer surface, which appears in layer $\alpha$ beneath that surface $D^{\alpha}$. We consider only bulklike bonded (that is, nonsurface) atoms in our analysis, and therefore consider the deformation and

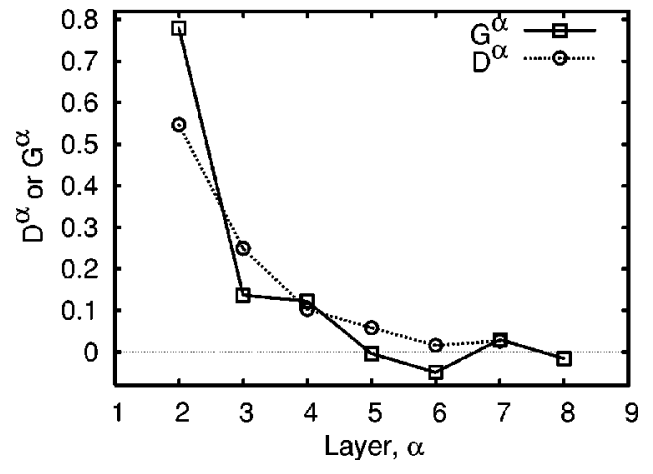

FIG. 5. Fraction of total local deformation appearing in layer $\alpha$ of a $c(4 \times 2)$ reconstructed surface slab $D^{\alpha}$ and fraction of total energy relaxation realized with the addition of Ge layer $\alpha$ to a $c(4 \times 2)$ reconstructed wetting layer slab $G^{\alpha}$.

energetics associated with the 2nd through 8th layers only, where the surface layer itself is layer 1 .

Figure 5 plots $D^{\alpha}$ and $G^{\alpha}$, as defined above. The correlation of these two quantities is consistent with, and thus supports, the interpretation of Tersoff ${ }^{26}$ that the variation of $\Gamma^{\mathrm{WL}}$ with thickness results primarily from the distribution of surface induced bond deformation. That is, that the reduction of wetting layer excess energy with increasing wetting layer thickness is a result of the transfer of surface induced deformation from the stiffer Si substrate to the softer Ge wetting layer. As most of the deformation resulting from the presence of the $c(4 \times 2)$ reconstructed surface appears in the first few layers beneath the surface-Fig. 5 shows that $\sim 80 \%$ of the considered deformation appears in layers two and three-we have strong variation in $\Gamma_{c(4 \times 2)}^{\mathrm{WL}}$ for wetting layers one, two, and three layers thick. The regime of steep decay in $\Gamma_{c(4 \times 2)}^{\mathrm{WL}}$ is correlated with the strongly deformed region of the wetting layer lying in the first few subsurface layers. The subsequent "settling" of $G^{\alpha}$ as $\Gamma^{\mathrm{WL}}$ converges to $\Gamma^{\mathrm{Ge}}$, is interpreted as resulting from weaker, longer-ranged components of the surface-induced strain field. This is taken to imply that the critical wetting layer thickness is primarily determined as the minimum film thickness for which the strong near-surface deformation lies within the wetting layer. With such an interpretation, the qualitative similarity between the $c(4 \times 2)$ and $4 \times 6$ curves in Figs. 3 and 4 suggests that the spatial extent of the high deformation region of the $4 \times 6$ DVL reconstructed surface is similar to that of $c(4 \times 2)$ surface.

Our current results, combined with the interpretation outlined above, provide a frame-work for predicting the qualitative behavior of the wetting layer energetics, and thus the wetting potential, for more complex surface structures. While further first-principles calculations are necessary to examine these structures in detail, we expect that the family of $4 \times N$ DVL reconstructed surfaces would have comparable distributions of bond deformation in the first few subsurface layers, and thus similar associated critical wetting-layer thicknesses. For $M \times N$ DVL patch reconstructions a slightly thicker band of high surface-induced deformation might be expected, leading to a slight increase in the critical wetting layer thickness. Due to the increased density of dimer vacancies in such a reconstruction, however, the effect on critical 
thickness, as measured in monolayers, would be reduced. Although our results provide no direct insight into the behavior of intermixed wetting layers, we expect that the relation between surface-induced bond deformation and wettinglayer energetics would hold also for alloyed films.

In summary, we have calculated the excess energy of the $\mathrm{Ge}$ on $\mathrm{Si}(100)$ wetting layer as a function of wetting layer thickness and surface reconstruction from first principles. For wetting layers at least 2 ML thick the $4 \times 6$ DVL superstructure of the $c(4 \times 2)$ alternating tilted dimer Ge $(100)$ surface reconstruction is stable with respect to the $c(4 \times 2)$ surface structure. An analysis of the relaxed atomic positions resulting from our calculations supports the view that the wetting layer excess energy dependence on thickness is related to the distribution of local bond deformation induced by the reconstructed wetting layer surface. ${ }^{26}$ The wetting layer excess energy decays rapidly as the wetting layer thickness increases from zero to approximately three layers. We interpret this steep decay as reflecting the shift of the near surface high bond deformation region from the Si substrate into the Ge overlayers. Further, our results provide an initial indication that this interpretation holds for the family of 4 $\times N$ DVL and $M \times N$ DVL patch reconstructions, where the distribution of the high bond deformation region is expected to be comparable to those of the reconstructions studied here. Finally, the qualitative form of the wetting layer excess energy dependence on wetting layer thickness calculated and discussed above represents a parametrization from first principles of the wetting potential as employed in continuum level modeling of the Ge on Si (100) system.

\section{ACKNOWLEDGMENTS}

This work was supported by the National Science Foundation under Program No. DMR-0102794, and made use of computing resources provided by NPACI at the University of Michigan. The authors also thank P. W. Voorhees for helpful discussions.
*Electronic address: beckm@northwestern.edu; URL: http:// cms.northwestern.edu/

${ }^{1}$ V. A. Shchukin and D. Bimberg, Rev. Mod. Phys. 71, 1125 (1999).

${ }^{2}$ I. Daruka and A.-L. Barabasi, Phys. Rev. Lett. 79, 3708 (1997).

${ }^{3}$ R. S. Williams, G. Medeiros-Ribeiro, T. I. Kamins, and D. A. A. Ohlberg, Annu. Rev. Physiol. 51, 527 (2000).

${ }^{4}$ D. J. Eaglesham and M. Cerullo, Phys. Rev. Lett. 64, 1943 (1990).

${ }^{5}$ Y.-W. Mo, D. E. Savage, B. S. Swartzentruber, and M. G. Lagally, Phys. Rev. Lett. 65, 1020 (1990).

${ }^{6}$ F. M. Ross, J. Tersoff, and R. M. Tromp, Phys. Rev. Lett. 80, 984 (1998).

${ }^{7}$ J. Tersoff, B. J. Spencer, A. Rastelli, and H. von Känel, Phys. Rev. Lett. 89, 196104 (2002).

${ }^{8}$ A. Rastelli, H. Von Känel, B. J. Spencer, and J. Tersoff, Phys. Rev. B 68, 115301 (2003).

${ }^{9}$ A. Vailionis, B. Cho, G. Glass, P. Desjardins, D. G. Cahill, and J. E. Greene, Phys. Rev. Lett. 85, 3672 (2000).

${ }^{10}$ P. Sutter and M. G. Lagally, Phys. Rev. Lett. 84, 4637 (2000).

${ }^{11}$ P. Sutter and M. G. Lagally, Mater. Sci. Eng., B 89, 45 (2002).

${ }^{12}$ R. M. Tromp, F. M. Ross, and M. C. Reuter, Phys. Rev. Lett. 84, 4641 (2000).

${ }^{13}$ A. A. Golovin, S. H. Davis, and P. W. Voorhees, Phys. Rev. E 68, 056203 (2003).

${ }^{14}$ H. R. Eisenberg and D. Kandel, Phys. Rev. Lett. 85, 1286 (2000).

${ }^{15}$ H. R. Eisenberg and D. Kandel, Phys. Rev. B 66, 155429 (2002).

${ }^{16}$ B. J. Spencer, Phys. Rev. B 59, 2011 (1999).

${ }^{17}$ M. Ortiz, E. A. Repetto, and H. Si, J. Mech. Phys. Solids 47, 697 (1999).

${ }^{18}$ L. G. Wang, P. Kratzer, M. Scheffler, and N. Moll, Phys. Rev. Lett. 82, 4042 (1999).

${ }^{19}$ A. A. Stekolnikov, J. Furthmüller, and F. Bechstedt, Phys. Rev. B 65, 115318 (2002).

${ }^{20}$ L. Spiess, A. J. Freeman, and P. Soukiassian, Phys. Rev. B 50,
2249 (1994).

${ }^{21}$ M. Needels, M. C. Payne, and J. D. Joannopoulos, Phys. Rev. Lett. 58, 1765 (1987).

${ }^{22}$ F. Liu, F. Wu, and M. G. Lagally, Chem. Rev. (Washington, D.C.) 97, 1045 (1997), and references therein.

${ }^{23}$ U. Kohler, O. Jusko, B. Muller, M. Hornvonhoegen, and M. Pook, Ultramicroscopy 42, 832 (1992).

${ }^{24}$ B. P. Uberuaga, M. Leskovar, A. P. Smith, H. Jónsson, and M. Olmstead, Phys. Rev. Lett. 84, 2441 (2000).

${ }^{25}$ L. Nurminen, F. Tavazza, D. P. Landau, A. Kuronen, and K. Kaski, Phys. Rev. B 68, 085326 (2003).

${ }^{26}$ J. Tersoff, Phys. Rev. B 43, 9377 (1991).

${ }^{27}$ J. Tersoff, Phys. Rev. B 45, 8833 (1992).

${ }^{28}$ X. Chen, F. Wu, Z. Zhang, and M. G. Lagally, Phys. Rev. Lett. 73, 850 (1994).

${ }^{29}$ C. V. Ciobanu, D. T. Tambe, and V. B. Shenoy, Surf. Sci. 556, 171 (2004).

${ }^{30}$ F. Liu and M. G. Lagally, Phys. Rev. Lett. 76, 3156 (1996).

${ }^{31}$ F.-K. Men and C.-R. Hsu, Phys. Rev. B 58, 1130 (1998).

${ }^{32}$ J. Oviedo, D. R. Bowler, and M. J. Gillan, Surf. Sci. 515, 483 (2002).

${ }^{33}$ K. Li, D. R. Bowler, and M. J. Gillan, Surf. Sci. 526, 356 (2003).

${ }^{34}$ G. Kresse and J. Hafner, Phys. Rev. B 47, 558 (1993).

${ }^{35}$ G. Kresse and J. Hafner, Phys. Rev. B 49, 14251 (1994).

${ }^{36}$ G. Kresse and J. Furthmüller, Comput. Mater. Sci. 6, 15 (1996).

${ }^{37}$ G. Kresse and J. Furthmüller, Phys. Rev. B 54, 11169 (1996).

${ }^{38}$ D. M. Ceperley and B. J. Alder, Phys. Rev. Lett. 45, 566 (1980).

${ }^{39}$ J. P. Perdew and A. Zunger, Phys. Rev. B 23, 5048 (1981).

${ }^{40}$ D. Vanderbilt, Phys. Rev. B 41, 7892 (1990).

${ }^{41}$ G. Kresse and J. Hafner, J. Phys.: Condens. Matter 6, 8245 (1994).

${ }^{42}$ S. Froyen, Phys. Rev. B 39, 3168 (1989).

${ }^{43}$ H. J. Monkhorst and J. D. Pack, Phys. Rev. B 13, 5188 (1976).

${ }^{44}$ A. Garcia and J. E. Northrup, Phys. Rev. B 48, 17350 (1993).

${ }^{45}$ J. E. Northrup, Phys. Rev. B 47, 10032 (1993). 